\title{
Aortic Stenosis: New complexity found in an "Old Friend" The clinical challenges of correct assessment and therapy of aortic stenosis
}

\author{
John D Rozich* \\ Tuomey Hospital Sumter, SC 29150, USA
}

\begin{abstract}
The evolution in understanding the pathophysiology and treatment of senile calcific aortic stenosis (AS) has mirrored many other age-related conditions. The development of enhanced imaging, novel delivery systems for valve replacement and rigorous outcomes trials examining optimal choices in therapy have all contributed to enhancement in the care of those impacted by this condition. Development and analysis of experimental models providing insights into etiology have also contributed to our improved understanding. Foreseeably, these advancements have allowed identification of multiple phenotypic presentations requiring a complete understanding to optimize outcomes. These choices mandate clarity as to the benefit, risk and outcomes and at times the subtleties that each potential option delivers must be examined in order to make reasonable clinical decisions. This manuscript is a focused effort to review the state-of-the-art assessment for correct AS diagnosis, classification and therapy as it now represents one of the most rapidly changing areas encountered in clinical practice.
\end{abstract}

\section{Introduction}

The last decade has witnessed a rather dramatic transformation in the care of patients suffering from senile calcific aortic stenosis (AS). Novel delivery systems for valve replacement have provided clinicians with choices between conventional surgical replacement and transcatheter aortic valve replacement (TAVR). But such therapeutic options have come at a time when a more detailed understanding of the hemodynamics of AS has also occurred. Using both human data and experimental models this more detailed appreciation of AS has illuminated a remarkable variability in pathological valvular presentations [1]. Conventional metrics used to identify and support clinical decision-making for valve replacement has become more nuanced [2,3]. Specifically, paradoxical "low flow-low gradient" (LFLG) severe AS with normal left ventricular ejection fraction (LVEF) has become an important if not confusing entity requiring meticulous assessment of both clinical and imaging details [4,5]. The current focused review is to highlight a practical approach in evaluating the severity of AS when presented with discordant metrics.

Clinicians must now be aware of a somewhat bewildering array of metrics that may support severe AS while appearing to be only moderate $[1,5]$. Failure to appreciate these variable presentations of AS may result in missed opportunities to optimally intervene and assist patients. Analysis of this entity of paradoxical LF-LG severe AS with normal LVEF raises several problematic concerns for clinicians and requires careful systematic evaluation $[1,5,6]$. First, as a general cautionary note, there is perhaps no other cardiac imaging procedure that is more dependent upon technician competence than routine echocardiography. This is not to diminish other fields or imaging modalities; it is simply a reflection of the tremendous influence that echocardiography technicians have over the finished product. Without skilled interrogation of valvular and cardiac pathology integrated with contemporaneous knowledge of requisite metrics, echocardiography may be inadequate or worse, lead to incorrect conclusions. Second, those interpreting the images must immediately be able to register potential sources of discordant metrics and either require additional focused imaging or explain the incongruous findings. It is therefore important to have a consistent approach in examining the metrics of AS.

\section{Traditional evaluation of aortic stenosis}

Clinicians commonly encounter AS in the elderly and historically had to answer two basic questions. First, how severe was the AS and second, did it require surgical replacement. Classic AS originally was graded by the invasive catheter-derived gradient between the left ventricle and the aorta. This invasive approach has evolved to a noninvasive assessment of valvular stenotic severity for several reasons $[7,8]$. First, as ultrasound technology advanced and echocardiography's application became more consistent, its rapid often bedside acquisition of valuable clinical metrics was validated thereby reducing catheter-based invasive valvular assessment [7,9]. Additionally, catheter assessment of valvular stenotic severity required left ventricular (LV) access which, often was technically challenging demanding careful positioning and advancing the catheter through an asymmetric calcified and stenotic orifice. This was done with a variety of catheters and wires, each with the potential to injure delicate adjacent structures [10].

While such complications were rare, studies emerged also demonstrating silent cerebral microembolism during retrograde catheterization of the left ventricle in patients with aortic stenosis

${ }^{\star}$ Correspondence to: John D Rozich, Tuomey Hospital Sumter, SC 29150, USA, E-mail: johnrozich@hotmail.com

Received: February 19, 2019; Accepted: March 22, 2019; Published: March 29, 2019 
[10-12]. Debate continues as to the relevant clinical outcome from these microembolic events, but ease of noninvasive assessment and elimination of a technically challenging if not potentially injurious procedure hastened the decline in use of catheter-based metrics for aortic stenosis [13]. Thus, over the last 25 years accurate noninvasive metrics provided by echocardiography within the clinical presentation of the patient's symptoms have become the more common practice. Typically, instantaneous velocities in severe AS were in excess of $4.0 \mathrm{~m} / \mathrm{s}$ with mean gradients greater that $40 \mathrm{mmHg}[3,14]$. This corresponds to a valve area of less than $0.8 \mathrm{~cm} 2$ when the mean gradient is greater than $40 \mathrm{mmHg}$. But it is well documented that aortic valve metrics are dependent on forward flow, which is itself linked to systolic performance and stroke volume (SV) $[2,3,5,14]$. Thus, reduced LVEF causing reduced forward flow and SV will reduce both transvalvular velocity and gradients. This is despite significant reduction in leaflet excursion and calcification of leaflet morphology.

\section{Reduced LVEF with low-flow and low-gradient}

The common approach to suspected severe AS but with LF-LG is to first assess the patient for decreased LVEF [15-18]. Actual reduction in the LVEF associated with a heavily calcified AV with reduced leaflet mobility may indicate severe AS, but could also represent moderate AS. The traditional approach is to perform a dobutamine infusion or even a dobutamine stress echocardiogram (DSE) to assess both LVEF and AV transvalvular metrics [15-18]. The use of dobutamine will often gradually recruit systolic performance and increase LVEF leading to enhanced flow across the AV. This dobutamine-dependent increased flow and velocity across the AV parallel an increased AV gradient and can unmask the actual severity of AS $[15,16]$. Increased dobutaminedependent systolic performance as measured by LVEF without significantly increased AV gradient support less severe AS. Thus, valve area calculations with dobutamine demonstrate an increase in flow but importantly if the previously mentioned only modest increase in the transaortic valve velocity occurs, this suggests less severe valve disease. This minimal impact on the valve gradient corresponds to a greater valve area and thus an inversely proportional grade of stenosis. These patients have less severe AS. Importantly also is a subset of patients who do not respond with an increase in LVEF of about $20 \%$ or greater in the face of careful dobutamine titration [19-21]. These patients are felt to "lack contractile reserve" or "lack of flow reserve". Their prognosis is poor despite surgical or medical therapy (Figure 1).

\section{Normal LVEF with low-flow and low-gradient}

But within the last decade a new presentation of severe AS has been recognized $[1,5]$. This manifests as severely calcified immobile AV leaflets but within the context of a normal LVEF and valve metrics that do not support classification of the AS being severe. This is the so-called "paradoxical LF-LG" with normal LVEF and severe AS [5]. Symptoms may also support severe AS, but the above metrics of transvalvular velocity and gradient obtained with adequate noninvasive imaging are not congruent. So, what are the explanations for these findings and do these findings actually represent severe AS?

\section{Metrics of flow: Ensuring accuracy}

The first issue is to ensure that the metrics of valvular flow are correct. While symptoms related to AS are pivotal in the overall strategy of care, clinicians routinely involved in monitoring AS progression often emphasize the ambiguities encountered in attempting to define subtle or changing symptoms potentially attributable to valvular heart disease. Thus, valid metrics are critical in the process of determining the severity of AS $[2,5,22]$. Incorrect measurements may account for reduced gradients, small aortic valve areas (AVA) and misclassification of moderate $\mathrm{AV}$ stenosis as severe when in fact it is moderate $[2,6,22,23]$. Early data suggested that parallel assessment of flow along the left ventricular outflow tract optimized the maximum flow and ensured accurate transvalvular measurements [24-26]. Placing the probe and measuring flow at angles wider than 30 degrees from the optimal parallel position may markedly reduce valvular velocity leading to underestimated gradients and overestimation of the AVA [27]. This would thus provide an underestimation of the AS severity. Clinicians must ensure that multiple windows are assessed for accurate maximum measurement of the velocity and gradient. Apical windows provide maximum Doppler velocities for the AV in only $40 \%$ of patients, while right parasternal windows reach only $50 \%$ for the maximum AV velocity [28]. The careful acquisition of multiple Doppler windows is important. A clinician should be concerned if low gradients exist, but limited windows are used, or if Doppler acquisition of velocities is technically difficult. These are hints that the metrics obtained may be inaccurate.

Additionally, when calculating AV valve areas, the precise dimension of the LV outflow tract (LVOT) must be obtained. Here deviations from the true LVOT may provide errors of either over, or underestimation of AS [29]. If the diameter of the LVOT is smaller than the true value,

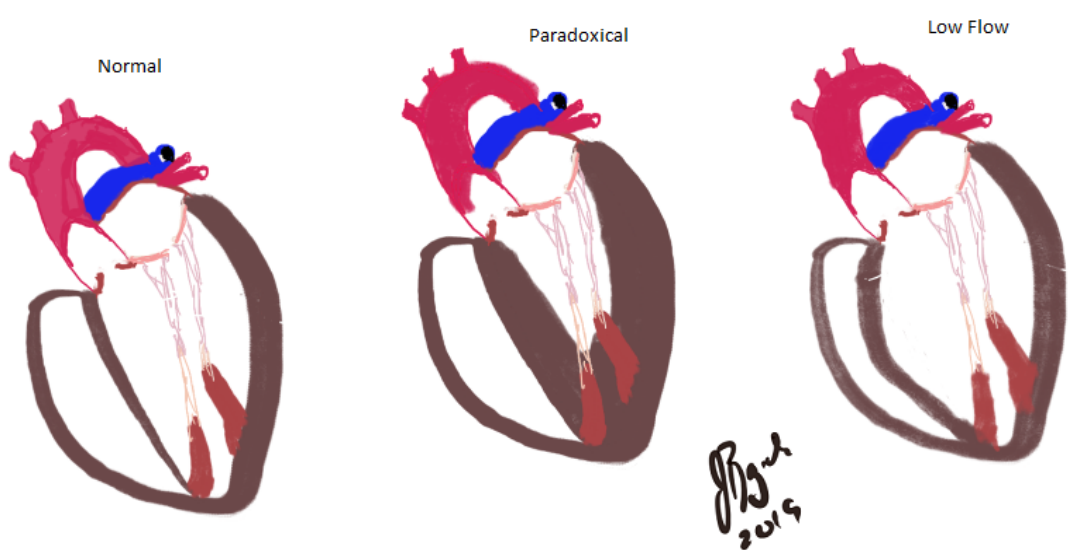

Figure 1. "Normal" Far left image is the typical HF-HG AS with normal LV cavity and normal LVEF. Center is the "Paradoxical LF-LG AS with small LV cavity and normal LVEF. Far right is the "low flow but LF-LG as often with dilated LV cavity and reduced LVEF also called "classical" low flow

HF: High flow, HG: High gradient; LV: Left ventricle; LVEF: Left ventricular ejection fraction; LF: Low flow; LG: Low gradient; AS: Aortic stenosis 
this leads to overestimation of the severity of AS. The LVOT diameter is a principal measurement in calculating the LVOT area and when it is combines with the LVOT time velocity integral (TVI) this provides the stroke volume at the level of the LVOT [30]. Importantly, the LVOT area uses the radius squared so that small changes in the diameter of the LVOT may magnify the error in the LVOT area and thus in the ultimate AVA since the denominator of the continuity equation is the TVI of the aortic transvalvular flow.

\section{AVA $=$ LVOT area $\times$ LVOT TVI}

\section{Aortic valve TVI}

Underestimation of the LVOT diameter may falsely lead to concluding that there is low-flow-low gradient severe AS when there is actually normal-flow and moderate AS. But subtle variations in the actual geometry of the LVOT may also influence calculations of LVOT area even when LVOT is correctly measured [30,31]. Recent studies have demonstrated that use of 2-dimensional (2-D) echo to measure the LVOT assumes a circular geometric shape [30]. Unfortunately, the actual shape of the LVOT is often elliptical and thus measurements using the assumed circular geometry may again introduce errors [32]. Existing data suggests that improper measurement of the LVOT area may be more pronounced when using 2-D echo if one measures the LVOT diameter $5-10 \mathrm{~mm}$ below the aortic annulus compared to measurements at the annulus [33]. Whether this report, or others that support a hybrid approach wherein LVOT diameter is calculated from multi-detector computed tomography (MDCT) or cardiac magnetic resonance (CMR) ultimately impacts clinical outcomes will await future confirmation. It must be acknowledged that each imaging modality has its own inherent limitations. For example, MDCT-Doppler (MDCT to accurately measure LVOT diameter-combined with conventional Doppler) was not superior to standard 2-D calculations for AVA determination. Thus, no single methodology or combination e.g. "hybrid approach" provides "perfect" data $[34,35]$.

Clinical assessment of a "typical case of severe AS" may rely on congruent data that "fit" expectations. Disparities between observed and expected metrics require detailed evaluation to ensure accuracy. But if metrics are validated what next? Is there a representative phenotype more frequently impacted with the "paradoxical low flow state"? This is a relevant question since estimates suggest that between $5-25 \%$ of all severe AS may present as paradoxical LF-LG in the setting of normal LVEF [36]. While other estimates suggest no more than $15 \%$ of patients present this way, it is important to appreciate our current understanding of the LF-LG normal LVEF phenomenon in severe AS [30].

\section{Confusing data}

A degree of confusion exists for clinicians in dealing with severe AS and is in part based on the variable management strategies, that continue to evolve, associated with LF-LG normal LVEF. It is important to emphasize that these outcomes are distinct from LF-LG AS with reduced $\operatorname{LVEF}(<40 \%)$. Thus, ambiguity may result when transparent and consistent segregation of hemodynamic variables is not properly addressed [37]. But it appears that paradoxical LF-LG AS (Normal LVEF) have worse outcomes compared with (a) high-gradient or (b) NF-LG AS, but better outcomes than classic LF-LG AS (reduced LVEF) [38]. However, with LF-LG normal LVEF studies support AV replacement since prognosis for even asymptomatic severe AS in the setting of LF-LG is poor [39-44]. Of note however, other studies suggest that these asymptomatic patients when re-examined actually have only moderate AS and achieve outcomes similar to other moderate AS patients with typical valvular metrics for moderate valvular pathology [42-44]. Again, data is limited but additional reports suggest that patients with LF-LG severe AS appear to have a poorer prognosis that those with severe AS and classic flow rate derived metrics [39]. It is therefore prudent to ask, "What variables may assist the clinician", in being suspicious of paradoxical LF-LG with normal LVEF causing an underestimation of the true or actual severity of AS?

\section{Is the patient hypertensive?}

Once the representative metrics have been confirmed, the next step is to ensure that the patient was not significantly hypertensive when these were acquired. Hypertension essentially may amplify the afterload mismatch that in effect blunts the valvular gradient by potentially impeding forward flow as blood exits the left ventricle [45-47]. It is as if the inertia of blood flow leaving the LV must overcome a significant impediment if the aortic pressure is elevated. This is in addition to the stenotic aortic valve that is already creating an impediment to blood flow from the LV. This concept is not inherently different from that supporting the traditional approach of minimizing the regurgitant volume in mitral insufficiency. Reducing afterload accomplished through systemic blood pressure reduction in effect reduces the barrier to blood leaving the left ventricle into the aorta, and as such proportionally increases flow out of the ventricle into the systemic circulation. The proportional increase into the systemic circulation is inversely related to regurgitant flow into the left atrium. Similarly, in paradoxical LF-LG severe AS, decreasing afterload alters the inherent mismatch identified when AS plus hypertension co-exist. This is the so called "afterload mismatch" and is worsened in patients when AS and hypertension together produce a significant impediment to forward flow out of the LV $[48,49]$.

Absent a hypertensive state, representative but confusing metrics suggesting paradoxical low flow should trigger a second critical question. Is there a typical phenotype that characterizes LF-LG normal LVEF severe AS? The literature suggests that while body mass index (BMI) or body surface area is not determinative in identifying paradoxical low flow severe AS, these metrics may serve as a trigger for heightened scrutiny $[30,36]$. A reduced BMI may correlate to a small heart and may support a more careful assessment for "paradoxical flow" in the context of incongruent AS metrics [30]. Finally, a small LV, thick LV walls and LV diastolic dysfunction seen on echocardiography should trigger suspicion of paradoxical flow.

But what exactly is meant by a small $L V$ ? Some authors infer that a LV end-diastolic dimension $<47 \mathrm{~mm}$ may be helpful [50]. In this setting if the LVEF is greater than $50 \%$ and the SV indexed to body surface area is $<35 \mathrm{ml} / \mathrm{m}^{2}$, these findings are consistent with low flow $[5,44]$. But importantly, direct measurements of LV dimension or volume alone are also inadequate markers for "paradoxical low flow". Here the surrogate of indexed flow to body surface area, provisionally corresponding to a small LV is more helpful [50]. There is also increased interest in the determination of "projected aortic valve area", which provides an estimate of the actual aortic valve area at "normal" flow, but this requires additional measurements, has its own inherent limitations (persistent discordance after DSE) and relies on calculations that again may introduce the potential of error [51]

\section{Diastolic heart failure in small hypertensive elderly women}

Heightened scrutiny of the small hypertensive elderly female with $\mathrm{LVH}$ has been proposed as the most likely subset of patients to carry 
this paradoxical low-flow variant of severe AS [30,50]. Of interest is that the elderly female with LVH is the same phenotype for diastolic heart failure or more specifically labeled heart failure with preserved EF, (HFpEF). Thus, authorities have noted that perhaps the paradoxical flow noted in LF-LG normal LVEF severe AS is another manifestation of altered diastolic hemodynamics $[1,4,5,23,30]$. Since the mean gradient and peak aortic velocity are dependent upon the magnitude of transvalvular flow, decreases in flow such as decreases in LVOT stroke volume may materially alter the gradient obtained across the AV. This is not artifactual, as it represents a true representative metric, but flow found in this circumstance is not "normal" flow.

Here the paradoxical LF-LG metrics are directly related to significant LV concentric remodeling with advanced diastolic dysfunction, impaired LV filling and reduced longitudinal systolic function $[1,4,5,30]$. The mechanistic explanation is that the actual volume filling the ventricle is reduced and to a variable and subtle degree, the popular use of global ejection fraction (EF) does not accurately reflect true LV systolic performance [36]. The result is that the LV accommodates a smaller volume, a smaller volume is ejected, this produces a reduced stroke volume (SV) and thus the reduction in SV represents low flow and the typical "velocities" produced in the setting of severe AS are not generated [1,36]. Clinicians understandably anticipate making therapeutic decisions based on metrics that satisfy guideline recommendations. Absent congruence between anticipated clinical progression and echo-derived metrics, decisions regarding management will be inconsistent in the setting of severe AS.

\section{Other factors}

Other factors may influence the reduced flow such as variability in diastolic filling intervals found in atrial fibrillation, mitral regurgitation and stenosis, right ventricular dysfunction, constrictive pericarditis and tricuspid regurgitation [2]. These latter variables do not represent the "paradoxical" low flow physiology. But these conditions introduce an often-dramatic beat to beat variability in the SV that superficially mimics altered flow found in the paradoxical or classical low flow conditions. The paradoxical low flow state for symptomatic severe AS must be recognized by the clinician by first becoming aware of typical clinical phenotypes associated with it. As discussed, the following may trigger clinical suspicion:

- Small Body Size or Small BMI

- Metrics that are not consistent or incongruent with clinical presentation.

- Pronounced LV concentric remodeling with advanced diastolic dysfunction.

- Impaired LV filling and reduced longitudinal systolic function.

- Suboptimal hypertensive management

- Co-existent mitral valvular disease

Encountering these suggestive elements in association with metrics that do not "fit" the clinical picture raises a reasonable need for careful examination of the calculations of aortic stenosis. Confirming that there is "paradoxical" flow with a normal LVEF versus "classic low flow state" associated with decreased LVEF is critical. To ensure that proper measurements of flow are obtained, the following will need to be reviewed:

- Proper parallel alignment of the Doppler to achieve maximum velocity of the aortic flow jet.
- Precision in the measurement of the LVOT diameter and thus the LVOT area.

- Confirming that the low-flow state has a stroke volume index of $<$ $35 \mathrm{ml} / \mathrm{m} 2[5,30,50]$.

- Confirming that measurements were taken absent a hypertensive state.

- Confirming that the transvalvular flow rate is $<200 \mathrm{ml} / \mathrm{s}$.

- In patients with small body size, AVA $>0.6 \mathrm{~cm} 2 / \mathrm{m} 2$ rules out severe AS $[5,30,50]$.

Somewhat advanced supplementary calculations are advocated to further discriminate between paradoxical and other low flow states, but likely will not be routinely employed in the "real world". But identifying paradoxical low flow is important since several studies and indeed metaanalyses have documented that in patients with classical LF-LG and those with paradoxical LF-LG, (with normal LVEF), have significantly enhanced survival benefit with AVR compared with conservative management $[1,5,30,36,43,50]$. Finally, the rapidly expanding TAVR indications may add some degree of urgency in the need for precision in determining aortic stenosis metrics. Originally restricted to patients judged to be at prohibitive risk for conventional AV surgery, TAVR use is now expanding into moderate risk populations and in Europe has entered the low and moderate-surgical-risk population [52-55].

\section{Final thoughts: questions regarding}

\section{Paradoxical LF-LG severe as normal LVEF}

Remaining questions exist as to the origins of the "paradoxical $L F-L G$ severe AS normal LVEF" phenotype. What accounts for the "preserved LVEF" but with low flow or reduced stroke volume? The aforementioned significant hypertrophy and small LV underwrite the proportional decrease in SV, but how does preserved LVEF and a reduced LV cavity form? Taking preserved LVEF first; this is likely interconnected to an insensitivity in systolic performance inherent in the LVEF. Simply stated, LVEF doesn't pick-up or show subtle forms of decreased LV systolic performance even though longitudinal myocardial function is often demonstrated to be impaired [36,56]. Further, reports have shown the LV myocardial fibrosis detected through cardiac MR late gadolinium enhancement and confirmed by biopsy may offer additional clues as to subtle but often overlooked source of reduced LV systolic performance despite "normal LVEF" [57].

Second, the finding that patients with low gradient but normal LVEF demonstrated the smallest LV cavity size and highest relative wall thickness showed both decreased longitudinal function and fibrotic noncontractile tissue at the subendocardium [57]. This latter combination of decreased longitudinal function is likely mechanistically related to the fibrotic noncontractile tissue at the subendocardium [57]. Again, the premise that some patients have a "hyper-hypertrophic" maladaptive response representing a phenotypic continuum in their responsiveness to load may even hint that the recognition of LFLG normal LVEF is temporally dependent; i.e. depending on when discovered or imaged. But it is inarguable that some patients manifest geometric changes in the LV cavity and wall structure causing attenuated flow in response to significant aortic stenosis while others develop the more common high flow states.

The underpinnings of the responsible cellular or molecular perturbations facilitating or directly causing the "hyper-hypertrophic" maladaptive response remain speculative. Again, it is thought that 
disproportionate LV growth or hypertrophy compromises LV volume in ways that result in recognized decreases in effective stroke volume [57]. Triggers for myocardial growth are a complex and nuanced interplay impacting myocardial cellular and molecular elements. Proposed factors, such as physical "load", or deformity of intact cellular length, surface area and sarcomere length have been shown sufficient to elicit induction of increased RNA and protein synthetic activity in adult mammalian cardiac cells [58]. As such, load may be determinative in recruiting early molecular signaling to induce a broader genomic activation leading to myocyte and interstitial hypertrophic growth. Thus, in this paradigm direct physical loading or pressure changes impacting myocardial structures represents the "trip wire" for cellular and molecular response and if sustained will geometrically alter the myocardium [59-62].

This structural hypertrophic change in the myocardium is ultimately seen as the small LV cavity-reduced stroke volume-low gradient with normal LVEF. But this begs a more fundamental question that centers on the molecular and cellular machinery responsible. Is the incongruent growth found explaining the "paradoxical" LF-LG phenotype actually a reflection of biological heterogeneity expressed at each level of the hypertrophic growth process? And thus, ultimate dissimilar physiology is the cumulative effect of potentially subtle but additive biologic changes each bending the final outcome until there is the recognizable variant (small hypertrophic LV cavity) distinct from the norm [63]. This heterogeneity in myocardial growth may also be selective in altering the typical ratios of myocyteinterstitial development, resulting in fibrotic subtypes characteristic of hypertrophic pathology and harming global cardiac performance $[64,65]$. Alternatively, is a single step, substantively altered, within the complex machinery of ventricular hypertrophic growth the principal culprit? Each of these are possibilities and are but two possibilities in a nearly infinite array of elusive or hidden deviations from the "normal" structural and functional mainstay.

It thus is foreseeable that segregation of the end-result of this remarkably complex growth response into essentially two recognized "LF-LG" phenotypes, (normal LVEF \& reduced LVEF), may oversimplify the biology of LV myocardial growth regulation. Exposure to varying loading conditions found in progressive calcific aortic stenosis may parallel an epigenetic phenomenon selectively recruiting dormant cellular or molecular systems in certain patients. Further, what is in effect a derivative outcome on LV growth is the linkage to disparate outcomes after three basic interventions (surgery, TAVR or medical therapy) $[37,43,50,65]$. It is again not surprising that this categorical construct is inadequate in our quest for an optimal therapeutic outcome. Ongoing investigation is addressing what variables are determinative as clinicians attempt to avoid the injudicious potential of oversimplifying the complex.

\section{Conclusion}

The previous era of conventional metrics used to identify and support clinical decision-making in the setting of severe AS is no more. The rather straightforward assessment of Doppler or catheter related pressure gradients used to quantify AV severity in the context of symptoms has become more refined if not more complex. Specifically, recognition of paradoxical LF-LG severe AS with normal LVEF challenges clinicians attempting to render appropriate management strategies for their patients. Importantly, this "paradoxical LF-LG" state must be distinguished from the classic LF-LG reduced LVEF as outcomes arguably may be different and the tools used examining deriving the actual severity of AS are also distinct. Careful integration of echoderived measurements within the context of the clinical presentation are essential. Additional forms of imaging or provocative testing may also be necessary. The often-subtle variances in each may provide clues as to the characteristic pathological valvular phenotype being addressed. Ultimately it is the judgement of experienced clinicians, and their attempt to ensure congruence between the expanding recognition of AS phenotypes and optimal therapeutic outcomes.

\section{References}

1. Pibarot P, Dumesnil JG (2013) Paradoxical low-flow, low-gradient aortic stenosis: new evidence, more questions. Circulation 128: 1729-1732. [Crossref]

2. Nishimura RA, Otto CM, Bonow RO (2014) AHA/ACC guideline for the managemen of patients with valvular heart disease: A report of the American College of Cardiology/ American Heart Association Task Force on Practice Guidelines. J Thorac Cardiovasc Surg 148: e1-e132.

3. Vahanian A, Alfieri O, Andreotti F (2012) Guidelines on the management of valvular heart disease the joint task force on the management of valvular heart disease of the european society of cardiology (esc) and the european association for cardio-thoracic surgery (EACTS). Eur J Cardiothorac Surg 42: 1-44.

4. Clavel MA, Berthelot-Richer M, Le Ven F (2015) Impact of classic and paradoxical low flow on survival after aortic valve replacement for severe aortic stenosis. $\mathrm{J} \mathrm{Am} \mathrm{Coll}$ Cardiol 65: 645-653.

5. Pibarot P, Clavel MA (2015) Management of paradoxical low-flow, low-gradien aortic stenosis: Need for an integrated approach, including assessment of symptoms, hypertension, and stenosis severity. J Am Coll Cardiol 65: 67-71.

6. Orwat S, Kaleschke G, Kerckhoff G, Radke R, Baumgartner H (2013) Low flow, low gradient severe aortic stenosis: Diagnosis, treatment and prognosis. EuroIntervention 9: $38-42$

7. Judge KW, Otto CM (1990) Doppler echocardiographic evaluation of aortic stenosis Cardiol Clin 8: 203-216.

8. Anger T, Bauer V, Plachtzik C (2014) Non-invasive and invasive evaluation of aortic valve area in 100 patients with severe aortic valve stenosis: Comparison of cardiac computed tomography with ECHO (transesophageal/transthoracic) and catheter examination. J Cardiol 63: 189-197.

9. Miller FA (2018) The integration of doppler ultrasound with two-dimensional echocardiography and the noninvasive cardiac hemodynamic revolution of the 1980s. $J$ Am Soc Echocardiogr 31: 1353-1365.

10. Leclercq F, Kassnasrallah S, Cesari JB (2001) Transcranial doppler detection of cerebral microemboli during left heart catheterization. Cerebrovasc Dis 12: 59-65.

11. Hamon M, Burzotta F, Oppenheim C (2007) Silent cerebral infarct after cardiac catheterization as detected by diffusion weighted magnetic resonance imaging: A randomized comparison of radial and femoral arterial approaches. Trials 8: 15.

12. Hamon M, Gomes S, Oppenheim C, Morello R, Sabatier R, et al. (2006) Cerebral microembolism during cardiac catheterization and risk of acute brain injury: A prospective diffusion-weighted magnetic resonance imaging study. Stroke 37: 20352038. [Crossref]

13. Scott DA, Evered LA, Gerraty RP, MacIsaac A, Lai-Kwon J, et al. (2014) Cognitive dysfunction follows left heart catheterisation but is not related to microembolic count Int J Cardiol 175: 67-71. [Crossref]

14. Hunt SA, Lytle BW, Nishimura R, Page RL, Riegel B, et al. (2006) ACC/AHA 2006 guidelines for the management of patients with valvular heart disease: A report of the american college of cardiology/american heart association task force on practice guidelines (writing committee to revise the 1998 guidelines for the management of patients with valvular heart disease): Developed in collaboration with the society of cardiovascular anesthesiologists: Endorsed by the society for cardiovascular angiography and interventions and the society of thoracic surgeons. Circulation 114: e84-231. [Crossref]

15. Deluche L, Laffort P, Pepin C, Vircoulon B, Bonnet J, et al. (1996) Value of echocardiography-dobutamine in the study of reserve myocardial contractility in aortic stenosis with alteration of left ventricular function. Apropos of a case. Ann Cardiol Angeiol 45: 523-526.

16. Grayburn PA (2006) Assessment of low-gradient aortic stenosis with dobutamine Circulation 113: 604-606. [Crossref] 
17. Monin JL, Guéret P (2003) Calcified aortic stenosis with left ventricular dysfunction and low transvalvular gradients. Must one reject surgery in certain cases? Arch Mal Coeur Vaiss 96: 864-870. [Crossref]

18. Monin JL, Gueret P (2005) Dobutamine hemodynamics for aortic stenosis with left ventricular dysfunction. Ann Cardiol Angeiol 54: 107-11.

19. Sathyamurthy I, Jayanthi K (2014) Low flow low gradient aortic stenosis: Clinical pathways. Indian Heart J 66: 672-677.

20. Subramanian H, Kunadian B, Dunning J (2008) Is it ever worth contemplating an aortic valve replacement on patients with low gradient severe aortic stenosis but poor left ventricular function with no contractile reserve? Interact Cardiovasc Thorac Surg 7: 301-305.

21. Tandon A, Grayburn PA (2013) Imaging of low-gradient severe aortic stenosis. JACC Cardiovasc Imaging 6: 184-195. [Crossref]

22. Annabi MS, Clisson M, Clavel MA, Pibarot P (2018) Workup and management of patients with paradoxical low-flow, low-gradient aortic stenosis. Curr Treat Options Cardiovasc Med 20: 49. [Crossref]

23. Ten Freyhaus H, Baldus S (2016) Paradoxical low-flow low-gradient aortic stenosis. Internist (Berl) 57: 317-322. [Crossref]

24. Berger M, Berdoff RL, Gallerstein PE, Goldberg E (1984) Evaluation of aortic stenosis by continuous wave Doppler ultrasound. J Am Coll Cardiol 3: 150-156. [Crossref]

25. Danielsen R, Nordrehaug JE, Vik-Mo H (1989) Factors affecting doppler echocardiographic valve area assessment in aortic stenosis. Am J Cardiol 63: 11071111. [Crossref]

26. Williams GA, Labovitz AJ, Nelson JG, Kennedy HL (1985) Value of multiple echocardiographic views in the evaluation of aortic stenosis in adults by continuouswave doppler. Am J Cardiol 55: 445-449. [Crossref]

27. Nishimura RA, Carabello BA (2012) Hemodynamics in the cardiac catheterization laboratory of the 21st century. Circulation 125: 2138-2150. [Crossref]

28. Thaden JJ, Nkomo VT, Lee KJ, Oh JK (2015) Doppler imaging in aortic stenosis: The importance of the nonapical imaging windows to determine severity in a contemporary cohort. J Am Soc Echocardiogr 28: 780-785.

29. Chin CW, Khaw HJ, Luo E (2014) Echocardiography underestimates stroke volume and aortic valve area: Implications for patients with small-area low-gradient aortic stenosis. Can J Cardiol 30: 1064-1072.

30. Clavel MA, Burwash IG, Pibarot P (2017) Cardiac imaging for assessing low-gradient severe aortic stenosis. JACC Cardiovasc Imaging 10: 185-202.

31. Khalique OK, Kodali SK, Paradis JM (2014) Aortic annular sizing using a novel 3-dimensional echocardiographic method: Use and comparison with cardiac computed tomography. Circ Cardiovasc Imaging 7: 155-163.

32. Clavel MA, Malouf J, Messika-Zeitoun D, Araoz PA, Michelena HI, et al. (2015) Aortic valve area calculation in aortic stenosis by CT and Doppler echocardiography. JACC Cardiovasc Imaging 8: 248-257.

33. LaBounty TM, Miyasaka R, Chetcuti S (2014) Annulus instead of LVOT diameter improves agreement between echocardiography effective orifice area and invasive aortic valve area. JACC Cardiovasc Imaging 7: 1065-1066.

34. Tsang W, Bateman MG, Weinert L, Pellegrini G, Mor-Avi V, et al. (2012) Accuracy of aortic annular measurements obtained from three-dimensional echocardiography, CT and MRI: Human in vitro and in vivo studies. Heart 98: 1146-1152. [Crossref]

35. Wang H, Hanna JM, Ganapathi A, Keenan JE, Hurwitz LM, et al. (2015) Comparison of aortic annulus size by transesophageal echocardiography and computed tomography angiography with direct surgical measurement. Am J Cardiol 115: 1568-1573. [Crossref]

36. Pibarot P, Dumesnil JG (2012) Low-flow, low-gradient aortic stenosis with normal and depressed left ventricular ejection fraction. J Am Coll Cardiol 60: 1845-1853.

37. Clavel MA, Fuchs C, Burwash IG, Mundigler G, Dumesnil JG, et al. (2008) Predictors of outcomes in low-flow, low-gradient aortic stenosis: Results of the multicenter TOPAS Study. Circulation 118: S234-242. [Crossref]

38. Clavel MA, Magne J, Pibarot P (2016) Low-gradient aortic stenosis. Eur Heart J 37: 2645-2657. [Crossref]

39. Connolly HM, Oh JK, Schaff HV, Roger VL, Osborn SL, et al. (2000) Severe aortic stenosis with low transvalvular gradient and severe left ventricular dysfunction: Result of aortic valve replacement in 52 patients. Circulation 101: 1940-1946. [Crossref]

40. Kulik A, Burwash IG, Kapila V, Mesana TG, Ruel M (2006) Long-term outcomes after valve replacement for low-gradient aortic stenosis: Impact of prosthesis-patient mismatch. Circulation 114: 553-558.
41. Levy F, Laurent M, Monin JL (2008) Aortic valve replacement for low-flow/lowgradient aortic stenosis operative risk stratification and long-term outcome: A European multicenter study. J Am Coll Cardiol 51: 1466-1472.

42. Jander N, Minners J, Holme I, Gerdts E, Boman K, et al. (2011) Outcome of patients with low-gradient "severe" aortic stenosis and preserved ejection fraction. Circulation 123: 887-895. [Crossref]

43. Clavel MA, Dumesnil JG, Capoulade R, Mathieu P, Senechal M, et al. (2012) Outcome of patients with aortic stenosis, small valve area, and low-flow, low-gradient despite preserved left ventricular ejection fraction. J Am Coll Cardiol 60: 1259-1267.

44. Hachicha Z, Dumesnil JG, Bogaty P, Pibarot P (2007) Paradoxical low-flow, lowgradient severe aortic stenosis despite preserved ejection fraction is associated with higher afterload and reduced survival. Circulation 115: 2856-2864. [Crossref]

45. Briand M, Dumesnil JG, Kadem L, Tongue AG, Rieu R, et al. (2005) Reduced systemic arterial compliance impacts significantly on left ventricular afterload and function in aortic stenosis: Implications for diagnosis and treatment. J Am Coll Cardiol 46: 291298. [Crossref]

46. Eleid MF, Nishimura RA, Sorajja P, Borlaug BA (2013) Systemic hypertension in low-gradient severe aortic stenosis with preserved ejection fraction. Circulation 128 : 1349-1353.

47. Kadem L, Dumesnil JG, Rieu R, Durand LG, Garcia D, et al. (2005) Impact of systemic hypertension on the assessment of aortic stenosis. Heart 91: 354-361. [Crossref]

48. Ross J (1976) Afterload mismatch and preload reserve: A conceptual framework for the analysis of ventricular function. Prog Cardiovasc Dis 18: 255-264.

49. Carabello BA (2013) Introduction to aortic stenosis. Circ Res 113: 179-185. [Crossref]

50. Pibarot P, Dumesnil JG (2010) Assessment of aortic stenosis severity: When the gradient does not fit with the valve area. Heart 96: 1431-1433.

51. Blais C, Burwash IG, Mundigler G (2006) Projected valve area at normal flow rate improves the assessment of stenosis severity in patients with low-flow, low-gradien aortic stenosis: The multicenter TOPAS (Truly or Pseudo-Severe Aortic Stenosis) study. Circulation 113: 711-721.

52. Mylotte D, Osnabrugge RLJ, Windecker S (2013) Transcatheter aortic valve replacement in Europe: Adoption trends and factors influencing device utilization. $J$ Am Coll Cardiol 62: 210-219.

53. Iturra SA, Suri RM, Greason KL, Stulak JM, Burkhart HM (2014) Outcomes of surgica aortic valve replacement in moderate risk patients: Implications for determination of equipoise in the transcatheter era. $J$ Thorac Cardiovasc Surg 147: 127-132. [Crossref]

54. Krasopoulos G, Falconieri F, Benedetto U (2016) European real-world trans-catheter aortic valve implantation: Systematic review and meta-analysis of European national registries. J Cardiothorac Surg 11: 150-159.

55. Khan SU, Lone AN, Saleem MA, Kaluski E (2017) Transcatheter vs surgical aorticvalve replacement in low- to intermediate-surgical-risk candidates: A meta-analysis and systematic review. Clin Cardiol 40: 974-981.

56. Mehrotra P, Jansen K, Flynn AW (2013) Differential left ventricular remodelling and longitudinal function distinguishes low flow from normal-flow preserved ejection fraction low-gradient severe aortic stenosis. Eur Heart J 34: 1906-1914.

57. Herrmann S, Stork S, Niemann M (2011) Low-gradient aortic valve stenosis myocardia fibrosis and its influence on function and outcome. J Am Coll Cardiol 58: 402-412.

58. Mann DL, Kent RL, Cooper Gt (1989) Load regulation of the properties of adult feline cardiocytes: Growth induction by cellular deformation. Circ Res 64: 1079-1090.

59. Cooper G, Kent RL, Mann DL (1989) Load induction of cardiac hypertrophy. J Mol Cell Cardiol 21: 11-30. [Crossref]

60. Jones LG, Rozich JD, Tsutsui H, Cooper (1992) Endothelin stimulates multiple responses in isolated adult ventricular cardiac myocytes. Am J Physiol 263: 1447-1454.

61. Kent RL, Rozich JD, McCollam PL, McDermott DE, Thacker UF, et al. (1993) Rapid expression of the $\mathrm{Na}(+)-\mathrm{Ca} 2+$ exchanger in response to cardiac pressure overload. $\mathrm{Am}$ J Physiol 265: H1024-1029. [Crossref]

62. Rozich JD, Barnes MA, Schmid PG, Zile MR, McDermott PJ, et al. (1995) Load effects on gene expression during cardiac hypertrophy. J Mol Cell Cardiol 27: 485-499. [Crossref]

63. Tagawa H, Rozich JD, Tsutsui H, Narishige T, Kuppuswamy D, et al. (1996) Basis for increased microtubules in pressure-hypertrophied cardiocytes. Circulation 93: 12301243. [Crossref] 
Rozich JD (2019) Aortic Stenosis: New complexity found in an "Old Friend” The clinical challenges of correct assessment and therapy of aortic stenosis

64. López B, González A, Ravassa S, Beaumont J, Moreno MU, et al. (2015) Circulating biomarkers of myocardial fibrosis: The need for a reappraisal. J Am Coll Cardiol 65 : 2449-2456. [Crossref]
65. Condorelli G, Jotti GS, Pagiatakis C (2016) Fibroblast senescence as a therapeutic target of myocardial fibrosis: Beyond spironolactone? J Am Coll Cardiol 67: 20292031 .

Copyright: (C2019 Rozich JD. This is an open-access article distributed under the terms of the Creative Commons Attribution License, which permits unrestricted use, distribution, and reproduction in any medium, provided the original author and source are credited. 